\title{
ON THE BITOPOLOGICAL EXTENSION OF THE BING METRIZATION THEOREM
}

\author{
SALVADOR ROMAGUERA and JOSEFA MARÍN
}

(Received 20 December 1984; revised 14 October 1985)

Communicated by J. H. Rubinstein

\begin{abstract}
Based on a Junnila's paracompactness characterization we give a definition of pairwise paracompact space which permits us to prove that a bitopological space is quasi-metrizable if, and only if, it is a pairwise developable and pairwise paracompact space. An easy consequence of this result is the biquasi-metric form of the Morita metrization theorem. We also give some results on open mappings and strong quasi-metrics.

1980 Mathematics subject classification (Amer. Math. Soc.): primary 54 E 35, 54 E 55; secondary 54 C 10, 54 D 18, 54 E 30.

Keywords and phrases: biquasi-metrization, bitopological space, open mapping, pairwise developable space, pairwise paracompact space, strong quasi-metric.
\end{abstract}

\section{Introduction}

Throughout this paper all topologies are $T_{1}$. There are several attempts to give an adequate definition of bitopological paracompactness (see, for example, [3], [13], [2], [8], [6]). Since every metrizable space is paracompact, it would be desirable for all biquasi-metrizable spaces to be pairwise paracompact. By using a Junnila's paracompactness characterization [7], we give a definition of bitopological paracompactness which verifies the above mentioned condition. With this and a suitable notion of bitopological development (Definitions 1,2 and 3) we prove a biquasi-metric analogue of a well-known theorem of Bing (a space is metrizable if, and only if, it is paracompact and developable [1]). We deduce a biquasi-metric similar of the Morita metrization theorem [12] as an easy corollary. We also obtain some results referring to open mappings about these class of spaces and, finally, we give some bitopological generalizations of theorems belonging to Stoltenberg [14] about strongly quasi-metrizable spaces.

(ㄷ) 1988 Australian Mathematical Society $0263-6115 / 88 \$ A 2.00+0.00$ 


\section{Quasi-metrization of pairwise developable spaces}

A quasi-metric on a set $X$ is a mapping $d$ from $X \times X$ into the real numbers $R$ satisfying for all $x, y, z \in X$ : (i) $d(x, y) \geqslant 0$, (ii) $d(x, y)=0$ implies $x=y$, (iii) $d(x, y) \leqslant d(x, z)+d(z, y)$. The topology $\tau(d)$ induced on $X$ by $d$ has as a base the family of balls $\left\{B_{d}(x, r): x \in X, r>0\right\}$ where $B_{d}(x, r)=\{y \in X: d(x, y)$ $<r\}$. Each quasi-metric $d$ induces a conjugate quasi-metric $d^{-1}$ defined by $d^{-1}(x, y)=d(y, x)$ for all $x, y \in X$. The biquasi-metrization problem is to find necessary and sufficient conditions for $\mathscr{P}=\tau(d)$ and $\mathscr{Q}=\tau\left(d^{-1}\right)$ for some quasi-metric $d$ on $X$, where $(X, \mathscr{P}, \mathscr{Q})$ is a bitopological space. A biquasi-metrizable space is a quasi-metrizable bitopological space.

In the following $N$ will denote the set of non-negative integer numbers.

DEFINITION 1. A pair open cover in a bitopological space $(X, \mathscr{P}, \mathscr{Q})$ is a family of pairs

$$
\left\{\left(G_{\alpha}, H_{\alpha}\right): \alpha \in I\right\}
$$

such that

(i) for each $\alpha \in I, G_{\alpha}$ is a $\mathscr{P}$-open set and $H_{\alpha}$ is a 2 -open set.

(ii) $\mathscr{G}=\left\{G_{\alpha}: \alpha \in I\right\}$ and $\mathscr{H}=\left\{H_{\alpha}: \alpha \in I\right\}$ are covers of $X$.

(iii) for each $x \in X$ there is an $\alpha \in I$ such that $x \in G_{\alpha} \cap H_{\alpha}$.

In the following we will denote the pair open cover $(1)$ in the form $(\mathscr{G}, \mathscr{H})$.

Let $(\mathscr{G}, \mathscr{H})$ and $\left(\mathscr{G}^{\prime}, \mathscr{H}^{\prime}\right)$ pair open covers of $(X, \mathscr{P}, \mathscr{Q})$. We say that $\left(\mathscr{G}^{\prime}, \mathscr{H}^{\prime}\right)$ refines $(\mathscr{G}, \mathscr{H})$ (that is, $\left(\mathscr{G}^{\prime}, \mathscr{H}^{\prime}\right)<(\mathscr{G}, \mathscr{H})$ ) if for each pair $\left(G_{\alpha}^{\prime}, H_{\alpha}^{\prime}\right) \in\left(\mathscr{G}^{\prime}, \mathscr{H}^{\prime}\right)$ there is a pair $\left(G_{\beta}, H_{\beta}\right) \in(\mathscr{G}, \mathscr{H})$ such that $G_{\alpha}^{\prime} \subset G_{\beta}$ and $H_{\alpha}^{\prime} \subset H_{\beta}$.

Definition 2. Let $(\mathscr{G}, \mathscr{H})$ be a pair open cover of $(X, \mathscr{P}, \mathscr{Q})$. Let $A$ be a non-empty subset of $X$. We define

$$
\operatorname{St}(A, \mathscr{G}, \mathscr{H})=\bigcup\left\{G_{\alpha} \in \mathscr{G}: A \cap H_{\alpha} \neq \varnothing\right\}
$$

and

$$
\operatorname{St}(A, \mathscr{H}, \mathscr{G})=\bigcup\left\{H_{\alpha} \in \mathscr{H}: A \cap G_{\alpha} \neq \varnothing\right\} .
$$

If $A=\{x\}, x \in X$, we write

$$
S t(x, \mathscr{G}, \mathscr{H})=\bigcup\left\{G_{\alpha} \in \mathscr{G}: x \in H_{\alpha}\right\}
$$

and

$$
S t(x, \mathscr{H}, \mathscr{G})=\bigcup\left\{H_{\alpha} \in \mathscr{H}: x \in G_{\alpha}\right\}
$$


Similarly, we define

and

$$
S t^{2}(x, \mathscr{G}, \mathscr{H})=\operatorname{St}(\operatorname{St}(x, \mathscr{G}, \mathscr{H}), \mathscr{G}, \mathscr{H})
$$

$$
S t^{2}(x, \mathscr{H}, \mathscr{G})=\operatorname{St}(\operatorname{St}(x, \mathscr{H}, \mathscr{G}), \mathscr{H}, \mathscr{G})
$$

Then, it is obvious that

$$
S t^{2}(x, \mathscr{G}, \mathscr{H})=\bigcup\{S t(y, \mathscr{G}, \mathscr{H}): y \in S t(x, \mathscr{G}, \mathscr{H})\}
$$

and

$$
S t^{2}(x, \mathscr{H}, \mathscr{G})=\bigcup\{S t(y, \mathscr{H}, \mathscr{G}): y \in S t(x, \mathscr{H}, \mathscr{G})\}
$$

Definition 3. A pair development in a bitopological space $(X, \mathscr{P}, \mathscr{Q})$ is a sequence $\left\{\left(\mathscr{G}_{n}, \mathscr{H}_{n}\right): n \in N\right\}$ of pair open covers of $X$ such that, for each $x \in X$,

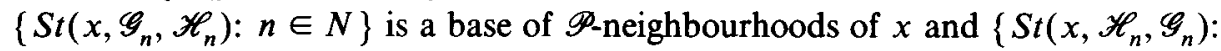
$n \in N\}$ is a base of 2 -neighbourhoods of $x$.

If $\left\{\left(\mathscr{G}_{n}, \mathscr{H}_{n}\right): n \in N\right\}$ is a pair development it is easy to see that, without loss of generality, we can take $\left(\mathscr{G}_{n+1}, \mathscr{H}_{n+1}\right)<\left(\mathscr{G}_{n}, \mathscr{H}_{n}\right)$ for each $n \in N$.

$(X, \mathscr{P}, \mathscr{Q})$ is pairwise developable if it has a pair developement $\left\{\left(\mathscr{G}_{n}, \mathscr{H}_{n}\right)\right.$ : $n \in N\}$ such that $\left(\mathscr{G}_{n+1}, \mathscr{H}_{n+1}\right)<\left(\mathscr{G}_{n}, \mathscr{H}_{n}\right)$ for each $n \in N$.

Remarks. (i) If in the above definition we put $\mathscr{P}=\mathscr{Q}$, then we have the concept of developable topological space.

(ii) Every bitopological quasi-metrizable space is a pairwise developable space.

Proposition 1. In a bitopological space $(X, \mathscr{P}, \mathscr{Q})$ are equivalent

(a) $(X, \mathscr{P}, \mathscr{Q})$ is pairwise developable,

(b) $(X, \mathscr{P}, \mathscr{Q})$ has a sequence $\left\{\left(\mathscr{G}_{n}, \mathscr{H}_{n}\right): n \in N\right\}$ of pair open covers such that

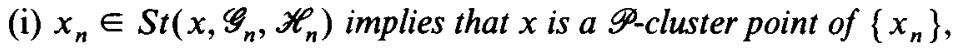

(ii) $x_{n} \in \operatorname{St}\left(x, \mathscr{H}_{n}, \mathscr{G}_{n}\right)$ implies that $x$ is a 2-cluster point of $\left\{x_{n}\right\}$.

(c) There are mappings $g: N \times X \rightarrow \mathscr{P}$ and $h: N \times X \rightarrow \mathscr{2}$ such that

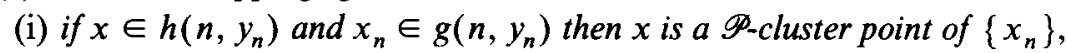

(ii) if $x \in g\left(n, y_{n}\right)$ and $x_{n} \in h\left(n, y_{n}\right)$ then $x$ is a 2-cluster point of $\left\{x_{n}\right\}$,

(iii) $x \in g(n, x) \cap h(n, x)$ for each $x$ and each $n$.

PROof. (a) implies (b). Let $U$ be a $\mathscr{P}$-neighbourhood of $x$. There is a $k \in N$ such that $\operatorname{St}\left(x, \mathscr{G}_{k}, \mathscr{H}_{k}\right) \subset U$. Then $x_{n} \in U$ for each $n \geqslant k$. This proves (i). Similarly we have (ii).

(b) implies (a). It is immediate supposing that condition (a) is not true.

(a) implies (c). It is enough to take, for each $x \in X$ and each $n \in N$, $g(n, x)=G_{\alpha}$ and $h(n, x)=H_{\alpha}$ such that $x \in G_{\alpha} \cap H_{\alpha}$ and $\left(G_{\alpha}, H_{\alpha}\right) \in\left(\mathscr{G}_{n}, \mathscr{H}_{n}\right)$.

(c) implies (a). It is enough to take, for each $n \in N, \mathscr{G}_{n}=\{g(n, x): x \in X\}$ and $\mathscr{H}_{n}=\{h(n, x): x \in X\}$. 
In [7] Junnila obtains the following characterization of paracompactness. A regular topological space $X$ is paracompact if, and only if, given an open cover $\mathscr{G}$ of $X$, then for each $x \in X$ there is a sequence $\left\{U_{n}(x): n \in N\right\}$ of neighbourhoods of $x$ such that:

(i) $y \in U_{n}(x) \Leftrightarrow x \in U_{n}(y)$.

(ii) if $x \in X$ there is an $n \in N$ and a $G \in \mathscr{G}$ with $U_{n}^{2}(x)=\bigcup\left\{U_{n}(y): y \in\right.$ $\left.U_{n}(x)\right\} \subset G$.

This result suggests the following definition.

DefinItION 4. A pairwise regular bitopological space $(X, \mathscr{P}, \mathscr{Q})$ is pairwise paracompact if given a pair open cover $(\mathscr{G}, \mathscr{H})$ of $(X, \mathscr{P}, \mathscr{Q})$ then for each $x \in X$ there is a sequence $\left\{U_{n}(x): n \in N\right\}$ of $\mathscr{P}$-neighbourhoods of $x$ and a sequence $\left\{V_{n}(x): n \in N\right\}$ of 2 -neighbourhoods of $x$ such that

(i) $y \in U_{n}(x) \Leftrightarrow x \in V_{n}(y)$.

(ii) if $x \in X$ there is an $n \in N$ and a pair $\left(G_{\alpha}, H_{\alpha}\right) \in(\mathscr{G}, \mathscr{H})$ with $U_{n}^{2}(x) \subset G_{\alpha}$ and $V_{n}^{2}(x) \subset H_{\alpha}$.

It is clear that every quasi-metrizable bitopological space is pairwise paracompact.

A local quasi-uniformity on a set $X$ is [11] a filter $\mathscr{U}$ on $X \times X$ such that:

(i) $\Delta \subset U$ for each $U \in \mathscr{U}$, where $\Delta=\{(x, x): x \in X\}$.

(ii) for each $x \in X$ and $U \in \mathscr{U}$ there is an $V \in \mathscr{U}$ such that $V^{2}(x) \subset U(x)$.

(iii) $\cap\{U: U \in \mathscr{U}\}=\Delta$

If $\mathscr{U}$ is a local quasi-uniformity on $X$ then $\tau(\mathscr{U})=\{A \subset X$ : for each $x \in A$ there is a $U \in \mathscr{U}$ with $U(x) \subset A\}$ is a topology for $X$ and $\mathscr{U}$ is called compatible with a topology $\tau$ on $X$ provided that $\tau=\tau(\mathscr{U})$.

The following result is a biquasi-metric extension of the famous Bing metrization theorem [1].

THEOREM 1. A bitopological space $(X, \mathscr{P}, \mathscr{2})$ is quasi-metrizable if, and only if, it is pairwise paracompact and pairwise developable.

Proof. Sufficient condition. By hypothesis there is a pair development $\left\{\left(\mathscr{G}_{n}, \mathscr{H}_{n}\right): n \in N\right\}$ such that $\left(\mathscr{G}_{n+1}, \mathscr{H}_{n+1}\right)<\left(\mathscr{G}_{n}, \mathscr{H}_{n}\right)$ for each $n \in N$. Given $\left(\mathscr{G}_{n}, \mathscr{H}_{n}\right)$, there is, for each $x \in X$, a sequence $\left\{U_{n m}(x): m \in N\right\}$ of $\mathscr{P}$-neighbourhoods of $x$ and a sequence $\left\{V_{n m}(x): m \in N\right\}$ of 2 -neighbourhoods of $x$ which satisfy the conditions (i), (ii) of Definition 4 . For each pair $(n, m)$ we consider the following subsets of $X \times X$ :

and

$$
U_{n m}=\left\{(x, y): x \in X, y \in U_{n m}(x)\right\}
$$

$$
V_{n m}=\left\{(x, y): x \in X, y \in V_{n m}(x)\right\}
$$


Then, $\left\{U_{n m}: n, m \in N\right\}$ is a base of a local quasi-uniformity $\mathscr{U}$ on $X$. In fact, if $x \in X$ and $j, k \in N$, there is a $n \in N$ such that $\operatorname{St}\left(x, \mathscr{G}_{n}, \mathscr{H}_{n}\right) \subset U_{j k}(x)$. Also there is a $m \in N$ and a pair $\left(G_{\alpha}, H_{\alpha}\right)$ in $\left(\mathscr{G}_{n}, \mathscr{H}_{n}\right)$ that verifies $U_{n m}^{2}(x) \subset G_{\alpha}$ and $V_{n m}^{2}(x) \subset H_{\alpha}$ such that $x \in G_{\alpha} \cap H_{\alpha}$. Hence:

$$
U_{n m}^{2}(x) \subset G_{\alpha} \subset S t\left(x, \mathscr{G}_{n}, \mathscr{H}_{n}\right) \subset U_{j k}(x)
$$

The above relation proves that $\tau(\mathscr{U})=\mathscr{P}$, too.

Similarly, $\left\{V_{n m}: n, m \in N\right\}$ is a base of a local quasi-uniformity $\mathscr{V}$ in $X$, such that $\tau(\mathscr{V})=2$. The condition (i) of Definition 4 allow us to conclude that $\mathscr{U}^{-1}=\mathscr{V}$ and applying [4, Theorem 7] or [10, Theorem 5] we have the conclusion.

The necessary condition is obvious.

We deduce easily the biquasi-metric extension of the Morita metrization theorem.

COROLlary. A bitopological space $(X, \mathscr{P}, \mathscr{2})$ is quasi-metrizable if, and only if, it has a pair development $\left\{\left(\mathscr{G}_{n}, \mathscr{H}_{n}\right): n \in N\right\}$ such that for each $x \in X$ $\left\{S t^{2}\left(x, \mathscr{G}_{n}, \mathscr{H}_{n}\right): n \in N\right\}$ and $\left\{S t^{2}\left(x, \mathscr{H}_{n}, \mathscr{G}_{n}\right): n \in N\right\}$ are, respectively, base of $\mathscr{P}$-neighbourhoods and base of 2-neighbourhoods of $x$.

Proof. It is obvious that the space is pairwise developable. Let $(\mathscr{G}, \mathscr{H})$ be a pair open cover of $(X, \mathscr{P}, \mathscr{Q})$. For each $x \in X$ and for each $n \in N$, we define:

$$
U_{n}(x)=\operatorname{St}\left(x, \mathscr{G}_{n}, \mathscr{H}_{n}\right)
$$

and

$$
V_{n}(x)=\operatorname{St}\left(x, \mathscr{H}_{n}, \mathscr{G}_{n}\right) .
$$

Then, it is easy to prove that the space is pairwise paracompact. Hence, by Theorem $1,(X, \mathscr{P}, \mathscr{Q})$ is quasi-metrizable. The converse is obvious.

\section{Open mappings}

Let be $\left(X, \mathscr{P}_{1}, \mathscr{Q}_{1}\right),\left(Y, \mathscr{P}_{2}, \mathscr{Q}_{2}\right)$ two bitopological spaces. A mapping $f$ of $\left(X, \mathscr{P}_{1}, \mathscr{Q}_{1}\right)$ onto $\left(Y, \mathscr{P}_{2}, \mathscr{Q}_{2}\right)$ is continuous (open) if $f:\left(X, \mathscr{P}_{1}\right) \rightarrow\left(Y, \mathscr{P}_{2}\right)$ and $f$ : $\left(X, \mathscr{Q}_{1}\right) \rightarrow\left(Y, \mathscr{2}_{2}\right)$ are continuous (open).

THEOREM 2. Let $\left(X, \mathscr{P}_{1}, \mathscr{Q}_{1}\right)$ be a pairwise developable space and $f:\left(X, \mathscr{P}_{1}, \mathscr{Q}_{1}\right)$ $\rightarrow\left(Y, \mathscr{P}_{2}, \mathscr{Q}_{2}\right)$ a continuous, open and finite to one mapping. Then $\left(Y, \mathscr{P}_{2}, \mathscr{Q}_{2}\right)$ is pairwise developable.

Proof. Let $\left\{\left(\mathscr{G}_{n}, \mathscr{H}_{n}\right): n \in N\right\}$ be a pairwise development of $\left(X, \mathscr{P}_{1}, \mathscr{Q}_{1}\right)$. For each $n \in N$ let $\mathscr{G}_{n}^{\prime}=\left\{f\left(G_{\alpha}\right): G_{\alpha} \in \mathscr{G}_{n}\right\}$ and $\mathscr{H}_{n}^{\prime}=\left\{f\left(H_{\alpha}\right): H_{\alpha} \in \mathscr{H}_{n}\right\}$. Then 
$\left\{\left(\mathscr{G}_{n}^{\prime}, \mathscr{H}_{n}^{\prime}\right): n \in N\right\}$ is a pairwise development on $\left(Y, \mathscr{P}_{2}, \mathscr{Q}_{2}\right)$. In fact, let us suppose that there is a $y \in Y$ and a sequence $\left\{y_{n}\right\} \subset Y$ such that $y_{n} \in$ $\operatorname{St}\left(y, \mathscr{G}_{n}^{\prime}, \mathscr{H}_{n}^{\prime}\right)$, but $y$ is not point of $\mathscr{P}_{2}$-cluster of $\left\{y_{n}\right\}$. Hence, there is a $\mathscr{P}_{2}$-neighbourhood of $y, V$, and a $k \in N$ such that $y_{n} \in(X-V) \cap S t\left(y, \mathscr{G}_{n}^{\prime}, \mathscr{H}_{n}^{\prime}\right)$ when $n \geqslant k$. For each $y_{n}$ there exist $\left(G_{\alpha}, H_{\alpha}\right) \in\left(\mathscr{G}_{n}, \mathscr{H}_{n}\right), z_{n} \in G_{\alpha}$ and $x_{n} \in H_{\alpha}$ such that $f\left(z_{n}\right)=y_{n}, f\left(x_{n}\right)=y$. Since $f$ is finite to one, there is an $x \in X$, with $f(x)=y$, and a subsequence $\left\{x_{n_{i}}\right\}$ such that $x_{n_{i}}=x$ for each $i$. Besides, $x$ is a $\mathscr{P}_{1}$-cluster point of $\left\{z_{n_{i}}\right\}$ and, because of $f$ being continuous, $y$ is a $\mathscr{P}_{2}$-cluster point of $\left\{f\left(z_{n_{i}}\right)\right\}$, obtaining a contradiction.

If in the above theorem we put $\mathscr{P}=\mathscr{Q}$ one has a result of Gittings [5].

A mapping $f: X \rightarrow Y$ is called $k$-to one if for each $y \in Y, f^{-1}(y)$ consists of exactly $k$ points in $X$.

THFOREM 3. Let $\left(X, \mathscr{P}_{1}, \mathscr{Q}_{1}\right)$ be a pairwise paracompact space and $f$ : $\left(X, \mathscr{P}_{1}, \mathscr{Q}_{1}\right) \rightarrow\left(Y, \mathscr{P}_{2}, \mathscr{Q}_{2}\right)$ a continuous, open and $k$-to one mapping. Then $\left(Y, \mathscr{P}_{2}, \mathscr{Q}_{2}\right)$ is pairwise paracompact.

Proof. Let $(\mathscr{A}, \mathscr{B})=\left\{\left(A_{\alpha}, B_{\alpha}\right): \alpha \in I\right\}$ be a pair open cover in $\left(Y, P_{2}, Q_{2}\right)$. Then $(\mathscr{G}, \mathscr{H})=\left\{\left(f^{-1}\left(A_{\alpha}\right), f^{-1}\left(B_{\alpha}\right)\right): \alpha \in I\right\}$ is a pair open cover in $\left(X, \mathscr{P}_{1}, \mathscr{Q}_{1}\right)$. For each $y \in Y$ let $f^{-1}(y)=\left\{x_{1}, \ldots, x_{k}\right\}$. For each $i=1, \ldots, k$, there is a sequence $\left\{U_{n}\left(x_{i}\right): n \in N\right\}$ of $\mathscr{P}_{1}$-neighbourhoods of $x_{i}$ and a sequence $\left\{V_{n}\left(x_{i}\right)\right.$ : $n \in N\}$ of $\mathscr{Q}_{1}$-neighbourhoods of $x_{i}$ which satisfy the conditions (i), (ii) of Definition 4. Now, let

$$
\begin{aligned}
& U_{n}^{\prime}(y)=\bigcap_{i=1}^{k} f\left(U_{n}\left(x_{i}\right)\right), \\
& V_{n}^{\prime}(y)=\bigcap_{i=1}^{k} f\left(V_{n}\left(x_{i}\right)\right) .
\end{aligned}
$$

It is immediate to prove that $z \in U_{n}^{\prime}(y) \Leftrightarrow y \in V_{n}^{\prime}(z)$.

We have now an $x_{j} \in X$ such that $f\left(x_{j}\right)=y$. There is an $n \in N$ and a pair $\left(f^{-1}\left(A_{\alpha}\right), f^{-1}\left(B_{\alpha}\right)\right)$ with $U_{n}^{2}\left(x_{j}\right) \subset f^{-1}\left(A_{\alpha}\right)$ and $V_{n}^{2}\left(x_{j}\right) \subset f^{-1}\left(B_{\alpha}\right)$. Hence, $\bigcap_{i=1}^{k} f\left(U_{n}^{2}\left(x_{i}\right)\right) \subset f\left(U_{n}^{2}\left(x_{j}\right)\right) \subset A_{\alpha}$ and $\bigcap_{i=1}^{k} f\left(V_{n}^{2}\left(x_{j}\right)\right) \subset f\left(V_{n}^{2}\left(x_{j}\right)\right) \subset B_{\alpha}$. Since $f$ is $k$-to one we deduce easily that $U_{n}^{\prime 2}(y) \subset \bigcap_{i=1}^{k} f\left(U_{n}^{2}\left(x_{i}\right)\right)$ and $V_{n}^{\prime 2}(y) \subset$ $\bigcap_{i=1}^{k} f\left(V_{n}^{2}\left(x_{i}\right)\right)$. Finally, since pairwise regularity is preserved by means of $k$-to one continuous open mappings we have the result.

We have, as an immediate consequence of Theorems 1,2 and 3. 
Corollary. Let $\left(X, \mathscr{P}_{1}, \mathscr{Q}_{1}\right)$ be a quasi-metrizable space, and $f:\left(X, \mathscr{P}_{1}, \mathscr{Q}_{1}\right) \rightarrow$ $\left(Y, \mathscr{P}_{2}, \mathscr{Q}_{2}\right)$ a continuous, open and $k$-to one mapping. Then $\left(Y, \mathscr{P}_{2}, \mathscr{Q}_{2}\right)$ is quasi-metrizable.

\section{Strong quasi-metrics}

Strong quasi-metric spaces defined by Stoltenberg [14], and studied with detail by Künzi [9], [10], are a class of developable spaces [14, Theorem 3.1]. In the bitopological case, we have

Definition 5. A bitopological space $(X, \mathscr{P}, \mathscr{Q})$ is pairwise strongly quasimetrizable if there are two quasi-metrics on $X, d$ and $d^{\prime}$, such that $\mathscr{P}=\tau(d) \subset$ $\tau\left(d^{\prime-1}\right)$ and $\mathscr{Q}=\tau\left(d^{\prime}\right) \subset \tau\left(d^{-1}\right)$.

Note that if $\mathscr{P}=\mathscr{Q}$ we obtain the definition of strongly quasi-metrizable topological space.

Proposition 2. Every pairwise strongly quasi-metrizable space $(X, \mathscr{P}, \mathscr{Q})$ is pairwise developable.

Proof. Let $d$ and $d^{\prime}$ quasi-metrics on $X$ such that $\mathscr{P}=\tau(d) \subset \tau\left(d^{\prime-1}\right)$ and $\mathscr{Q}=\tau\left(d^{\prime}\right) \subset \tau\left(d^{-1}\right)$. For each $n \in N$ let

$$
\mathscr{G}_{n}=\left\{B_{d}\left(x, 2^{-n}\right): x \in X\right\}
$$

and

$$
\mathscr{H}_{n}=\left\{B_{d^{\prime}}\left(x, 2^{-n}\right): x \in X\right\} .
$$

We see that $\left\{\left(\mathscr{G}_{n}, \mathscr{H}_{n}\right): n \in N\right\}$ is a pair development. In fact, let $x \in X$ and let $U$ be a $\mathscr{P}$-neighbourhood of $x$. There are $m, n \in N$ with $m-1 \geqslant n$ such that

$$
B_{d^{\prime}-1}\left(x, 2^{-m}\right) \subset B_{d}\left(x, 2^{-(n+1)}\right) \subset B_{d}\left(x, 2^{-n}\right) \subset U .
$$

Then, $\operatorname{St}\left(x, \mathscr{G}_{m}, \mathscr{H}_{m}\right)=\bigcup\left\{B_{d}\left(y, 2^{-m}\right): x \in B_{d^{\prime}}\left(y, 2^{-m}\right)\right\} \subset U$ because if $p \in$ $B_{d}\left(y, 2^{-m}\right)$ and $x \in B_{d^{\prime}}\left(y, 2^{-m}\right)$ we have $d(x, p)<2^{-n}$ and, hence, $p \in U$.

Similarly we deduce that $\left\{\operatorname{St}\left(x, \mathscr{H}_{n}, \mathscr{G}_{n}\right): n \in N\right\}$ is base of 2 -neighbourhoods of $x$.

Stoltenberg proves in [14] that a topological space is metrizable if, and only if, it is paracompact and strongly quasi-metrizable. In this direction we obtain

THEOREM 4. A space $(X, \mathscr{P}, \mathscr{Q})$ is quasi-metrizable if, and only if, it is pairwise paracompact and pairwise strongly quasi-metrizable. 
Proof. For the sufficient condition apply Theorem 1 and Proposition 2. The necessary condition is obtained doing $d^{\prime}=d^{-1}$ when $\tau(d)=\mathscr{P}$ and $\tau\left(d^{-1}\right)=\mathscr{Q}$.

The proof of the following elementary remark is omitted.

Lemma. A bitopological space $(X, \mathscr{P}, \mathscr{Q})$ is pairwise developable if, and only if, there are mappings $g: N \times X \rightarrow \mathscr{P}$ and $h: N \times X \rightarrow 2$ such that

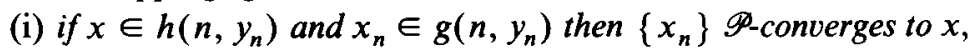

(ii) if $x \in g\left(n, y_{n}\right)$ and $x_{n} \in h\left(n, y_{n}\right)$ then $\left\{x_{n}\right\}$ 2-converges to $x$,

(iii) $x \in g(n, x) \cap h(n, x)$ for each $x$ and each $n$.

The following is a definition due to R. E. Hodel.

A topological space $(X, \mathscr{P})$ is a $\gamma$-space if there exists a mapping $g: N \times X \rightarrow \mathscr{P}$

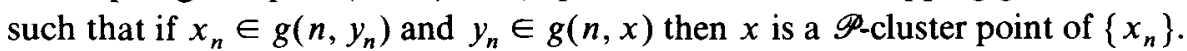

A bitopological space $(X, \mathscr{P}, \mathscr{Q})$ is a bi- $\gamma$ space if $(X, \mathscr{P})$ and $(X, \mathscr{Q})$ are $\gamma$-spaces. Given a bitopological space $(X, \mathscr{P}, \mathscr{Q}) \mathscr{P} \vee \mathscr{Q}$ denotes the supremum topology of $\mathscr{P}$ and 2.

In [10] Künzi proves that a topological space is strongly quasi-metrizable if it is developable and $\gamma$. Here we have

THEOREM 5. Let $(X, \mathscr{P}, \mathscr{2})$ be a pairwise developable bi- $\gamma$ space, then $(X, \mathscr{P} \vee \mathscr{Q})$ is strongly quasi-metrizable.

Proof. Applying the above lemma it is easy to see that $(X, \mathscr{P} \vee \mathscr{Q})$ is a developable space. Also it is clear that $(X, \mathscr{P} \vee \mathscr{Q})$ is a $\gamma$-space. The Künzi theorem concludes the proof.

\section{Additional remarks}

The relation and differences between several notions of bitopological paracompactness, which appear in the literature, and the Definition 4 is clear. If $(X, \mathscr{P}, \mathscr{2})$ is pairwise Hausdorff and pairwise paracompact, in the sense of [2], [3] or [13], then $\mathscr{P}=\mathscr{2}$ and, obviously, $(X, \mathscr{P}, \mathscr{2})$ satisfies Definition 4 . On the other hand, if $(X, \mathscr{P}, \mathscr{Q})$ is quasi-metrizable with $\mathscr{P} \neq \mathscr{Q}$, then this space satisfies the Definition 4 but it is not pairwise paracompact in the sense of [2], [3] or [13].

The referee has noticed to the authors the existence of a new definition of bitopological paracompactness due to T. G. Raghavan and I. L. Reilly in 'A new bitopological paracompactness' (to appear): a bitopological space $(X, \mathscr{P}, \mathscr{Q})$ is $\delta$-pairwise paracompact if every open cover of $X$ by sets in $\mathscr{P}(\mathscr{2})$ has a $\mathscr{P} \vee \mathscr{Q}$-locally finite refinement by sets in $\mathscr{P} \vee \mathscr{Q}$. It is clear that every bitopological quasi-metrizable space is $\delta$-pairwise paracompact, however we don't know the 
relationship between this concept of bitopological paracompactness and the our one.

After this paper had been submitted for publication, L. M. Brown informed the authors that the notion of pairwise developable space also appears, in terms of dual covers, in his paper 'A topic in the theory of bitopological spaces' (Karademiz Univ. Math. J. 5 (1982), 142-149). It is easy to see the equivalence between Brown's definition and our definition of pairwise developable space.

The authors would like to thank Professor Brown for his cooperation and his information about this work. We are also grateful for the suggestions of the referee.

\section{References}

[1] R. H. Bing, 'Metrization of topological spaces', Canad. J. Math. 3 (1951), 175-186.

[2] M. C. Datta, 'Paracompactness in bitopological spaces and an application to quasi-metric spaces', Indian J. Pure Appl. Math. 8 (1977), 685-690.

[3] P. Fletcher, H. B. Hoyle III and C. W. Patty, 'The comparison of topologies', Duke Math. J. 36 (1969), 325-331.

[4] R. Fox, On metrizability and quasi-metrizability (preprint).

[5] R. F. Gittings, 'Finite-to-one open maps of generalized metric spaces', Pacific J. Math. 59 (1975), 33-41.

[6] A. Gutiérrez, On pairwise paracompact spaces (preprint).

[7] H. J. K. Junnila, Covering properties and quasi-uniformities of topological spaces (Thesis, Virginia Polytechnic Institute and State University, Blacksburg 1978).

[8] Ch. Konstandilaki-Savopoulou and I. L. Reilly, 'On Datta's bitopological paracompactness', Indian J. Pure Appl. Math. 12 (1981), 799-803.

[9] H. P. A. Künzi, Quasi-metrisierbare Räume (Thesis, Universität Bern, 1981).

[10] H. P. A. Künzi 'On strongly quasi-metrizable spaces', Arch. Math. (Basel) 41 (1983), 57-63.

[11] W. F. Lindgren and P. Fletcher, 'Locally quasi-uniform spaces with countable bases', Duke Math. J. 41 (1974), 231-240.

[12] K. Morita, 'A condition for the metrizability of topological spaces and for $n$-dimensionality', Sci. Rep. Tokyo Kyoiku Daigaku, Sect. A 5 (1955), 33-36.

[13] T. G. Raghavan and I. L. Reilly, 'Metrizability of quasi-metric spaces', J. London Math. Soc. 15 (1977), 169-172.

[14] R. A. Stoltenberg, 'On quasi-metric spaces', Duke Math. J. 36 (1969), 65-71.

Departamento de Matemática Pura

ETSICCP

Universidad Politécnica

46022 Valencia

Spain 